\title{
Health Professional Card der Schweizer Ärzteschaft ante portas
}

Max Giger, Judith Wagner

Korrespondenz:

Dr. med. Max Giger

Mitglied Zentralvorstand

Generalsekretariat FMH

CH-3000 Bern 15

max.giger@hin.ch
Nachdem die Ärztekammer im Dezember 2005 den Grundsatz und im Dezember 2006 ein Projekt zur Schaffung eines physischen und elektronischen Ausweises für alle in der Schweiz lebenden Ärztinnen und Ärzte beschlossen hatte, leitete der Zentralvorstand der FMH die Ausführung des Projektes «Health Professional Card» (HPC) ein. Die Abteilung E-Health der FMH wurde mit der Durchführung des Projektes beauftragt. Ein Steuerungsausschuss unter Beizug eines externen Projektcontrollers sowie ein technischer Experte begleiten das Projekt, so dass im Mai 2008 allen in der Schweiz lebenden bzw. beruflich aktiven Ärztinnen und Ärzten eine adäquate und sichere elektronische Identität im wachsenden E-Health-Bereich ausgehändigt werden kann. Die Schweiz folgt damit den Nachbarstaaten Deutschland und Österreich.

\section{Was kann die HPC?}

Die HPC identifiziert die darauf genannte Person als Ärztin oder Arzt. Durch die Personendaten und ein Foto, die durch eine sogenannte Registrierungsstelle (u.a. Notariat/Gemeinde, zertifizierte Institution) beglaubigt wurden, wird die eindeutige Identifikation der auf der HPC genannten Person sichergestellt. Es entsteht ein «Ausweis» der diesen Namen verdient und vom Sicherheitsniveau her den Möglichkeiten, die er eröffnet, entspricht. Die Möglichkeiten für einen Missbrauch, namentlich beim Bezug von Arzneimitteln in einer Apotheke, sind stark verringert.

Auf den Chip der HPC können verschiedene Schlüssel, sogenannte fortgeschrittene und qualifizierte Zertifikate, geladen werden. Diese Zertifikate, können von der Karte weder exportiert noch entwendet werden; sie bleiben damit immer bei der die HPC tragenden Person. Diese elektronischen Schlüssel werden u.a. von Microsoft- und Apple-basierenden Anwendungen als vertrauenswürdig erkannt und ermöglichen den sicheren elektronischen Verkehr unabhängig vom Provider. Sie garantieren einen sicheren, elektronischen und authentischen Austausch von vertraulichen oder rechtlich relevanten Daten mit anderen Personen und Institutionen im Gesundheitswesen.

Die «fortgeschrittenen Zertifikate» ermöglichen den elektronischen Datenaustausch mit
Personen und Institutionen, die deren Trägern den Zugang in ihr System erlauben. Mithilfe der Schlüssel auf dem Chip können Daten verschlüsselt und auch ausserhalb geschlossener Gemeinschaften (closed user group CUG) unabhängig vom Provider ausgetauscht werden. Die Zertifikate werden nicht auf einem zentralen Server gespeichert, was deren möglichen Missbrauch ausschliesst.

Die «qualifizierten Zertifikate» erlauben die rechtssichere elektronische Unterzeichnung eines elektronischen Dokumentes (u.a. Rezept zum Bezug von Arzneimitteln, Berichte von Radiologen, Pathologen, Verantwortlichen von Laboratorien).

\section{Was profitieren ich und die Patientinnen und Patienten von der HPC?}

Ausserhalb meiner direkten Berufstätigkeit kann ich mich mit der HPC als Ärztin oder Arzt ausweisen. Ich kann von jedem PC mit Kartenlesegerät unabhängig von meinem Aufenthalt sicher mit meinen Partnern im Gesundheitswesen kommunizieren und auf Datenbanken zugreifen.

Im Rahmen meiner ärztlichen Berufsausübung innerhalb einer Institution kann ich entsprechend dem Ausbaustandard der Software persönlich von jedem PC innerhalb und ausserhalb derselben - Kartenlesefähigkeit vorausgesetzt - Dokumente einsehen, bearbeiten und signieren. Während für die Einsicht in Patientenakten fortgeschrittene Zertifikate genügen, werden aus Sicherheitsgründen für deren Unterzeichnung in zunehmendem Mass qualifizierte Zertifikate eingesetzt werden. Ich kann als verantwortliche Kaderärztin oder verantwortlichen Kaderarzt die Patientenakten auch von ausserhalb des Spitals zu jeder Tages- und Nachtzeit einsehen und Entscheidungen für Patientinnen und Patienten aufgrund meines persönlichen Aktenstudiums mittragen. Ich kann im Idealfall die gesamte Bilddokumentation wenige Minuten nach deren Generierung einsehen. Diese Art der elektronischen Kommunikation liegt vollumfänglich im Interesse der Patientensicherheit, da so Schnittstellen reduziert und allfällige Missverständnisse ab ovo verhindert werden können. Die Suche von Literatur zur Lösung 
von aktuellen Problemen über Datenbanken und Bibliotheken mit eingeschränktem Zugriff wird ebenfalls unabhängig von Ort und Zeit.

Im Rahmen meiner hausärztlichen Berufsausübung kann ich innerhalb eines von den Spitälern im Einvernehmen mit den Patientinnen und Patienten definierten Bereichs einerseits Einsicht nehmen in deren Akten und andererseits rechtzeitig ohne (menschliche) Schnittstelle über die verordneten Massnahmen und Arzneimittel direkt bei deren Rückkehr nach Haus orientiert werden. Ich kann meine Befunde, Einweisungsschreiben und Fragen an Spitalärztinnen und Spitalärzte sowie Fachärztinnen und Fachärzte senden, unabhängig von Ort und Zeit. Ich kann im Einverständnis mit den Patientinnen und Patienten - bei Polymedikation selbstverständlich nach elektronischem Interaktionscheck [1] - signierte Rezepte an Apotheken senden und so im Interesse der Patientensicherheit allfällige Lesefehler verhindern.

\section{Wie beeinflusst die HPC die Gesundheitskosten?}

Durch die gesicherte elektronische Kommunikation innerhalb der Institutionen und vor allem zwischen den verschiedenen Leistungserbringern, aber auch mit den Leistungsträgern können Schnittstellen reduziert und die Übermittlungsprozesse effizienter gestaltet werden. Das Berichtswesen kann aufs wesentliche reduziert werden; insbesondere kann auf das Versenden von Labordaten und Röntgenfilmen [2] verzichtet werden. Berichte müssen nur einmal erstellt werden und können danach sicher an alle involvierten Personen versandt werden.

Auch Verordnungen von Arzneimitteln müssen nur einmal dokumentiert werden, dann können Checks durchgeführt sowie Rezepte generiert, signiert und sicher versandt werden. Fehler bei der Übermittlung von Berichten und Rezepten, die heute noch zu Komplikationen und Hospitalisationen führen und dadurch hohe Kosten generieren, können verhindert werden.

\section{Was haben HPC und Versichertenkarte miteinander gemeinsam?}

Im Prinzip ausser dem ersten Anblick nicht viel. Sollte jedoch die Versichertenkarte auch einen entsprechenden Chip (und Zertifikate) enthalten, kann die HPC-Trägerin oder der HPC-Träger autorisiert werden, auf die Daten auf der Karte oder vielleicht einmal sogar auf Patientendaten in Institutionen und Praxen zuzugreifen.

\section{Konklusion}

Durch die baldige Einführung der HPC wird die Kommunikation innerhalb des Gesundheitswesens effizienter und die Patientensicherheit erhöht. Eine schnelle Verfügbarkeit der HPC wird eine Katalysatorwirkung für konkrete E-Health-Anwendungen haben.

\section{Literatur}

1 Giger M. Ärztliche Berufsbildung und Patientensicherheit. Schweiz Ärztezeitung. 2007;88(29/30): 1239

2 Lipphardt N, Müller R, Römpler M, Kubik-Huch R Ein halbes Jahr filmloses Spital in Baden. Schweiz Ärztezeitung. 2007;88(26):1152-4. 